\title{
Improved Performance of a Compact 2-Element Microstrip Patch Antenna Array using a Novel Class of Corrugated Structures
}

\author{
Zuhair M. Hejazi and Asem S. Al-Zoubi \\ Department of Telecommunication Engineering, Hijjawi Faculty for Engineering Technology, \\ Yarmouk University, Irbid, Jordan, hejazi@yu.edu.jo, +962-2-7211111
}

\begin{abstract}
Microstrip patch antennas are very often used in array configurations. A novel corrugated structure of a single microstrip patch is investigated first. Very promising results were achieved. However, the isolation coupling issue, in particular and the antenna performance in general, cannot be verified with a single patch. Thus, in this research, a probe-fed 2-element patch array is investigated in detail using the proposed novel corrugated structures with various shapes and depths to explore the various effects on the isolation coupling in particular, resonant frequency and the gain, without changing the patch separation and consequently, the array size at all but using only the effects of the proposed novel corrugated structures. These effects are also, studied and reflected on the overall performance of such array. A significant reduction of the undesired isolation coupling is achieved with impressive improvements of the cross-polarization levels and somewhat the gain. The novel structures would also provide further miniaturization of the array by shifting down the center frequency with maintaining the same array size. This technique can be applied as a building block for multi-element array in the E-plane and H-plane microstrip patch array arrangements. Full-wave analysis simulations with HFSS and Sonnet commercial softwares are used in the investigations.
\end{abstract}

Key words: Microstip, patch antenna array, corrugated structures, isolation coupling, fringing effect, cross polarization

\section{INTRODUCTION}

Microstrip (MS) patch antennas are known to be suitable for aircraft, space craft and missiles as they do not interfere with the aerodynamic functions of these vehicles. Arrays of MS antennas can be conformal to wing shape or missiles. They also become popular for commercial mobile and wireless communication systems such as in pagers, cellular phones and base stations.

In general, their low profile, low radar cross-section, robustness, reconfigurability, compatibility with other planar circuit designs, low cost production and reliability make them attractive for continuous improvements and intensive research on single layer, dual or multiband antennas by Lee et al. (2009), Li et al. (2014), Sharma and Sharma (2014), Raj and Chauhan (2104), Casu et al. (2014), Khan et al. (2015), Singh et al. (2015) and Gour et al. (2017) as well as stacked micrositip patch antennas by Zakaria et al. (2012) and Kumar et al. (2016). The focus of the researchers has been to overcome various drawbacks of this type of antennas such as their low efficiency (due to dielectric and conductor losses), narrow frequency bandwidth and relatively low power capability. MS patch antennas at UWB for wireless applications using square, circle and hexagonal shapes have been studied by Rao and Prasad (2019).

Fringing effect and the isolation coupling: Surface waves are known to be excited and travel within the substrate and can be excited at the dielectric-to-air interface. They give rise to end-fire radiation but usually are undesired because they lead to undesired coupling between array elements. They, however are strongly dependent on $\varepsilon_{\mathrm{r}}$ and the thickness $h$ of the substrate. They increase as the substrate thickness h increases. Transmission Line Model (TLM) is the simplest of all. It represents the rectangular patch as a parallel-plate transmission line connecting two radiating slots, each has a width $\mathrm{W}$ and height $\mathrm{h}$ as shown in Fig. 1 where the propagation direction is $\mathrm{z}$.

The slots shown (\#1 and \#2) represent very high impedance terminations from both sides of the transmission line, i.e., almost an open circuit. This structure have highly resonant characteristics depending crucially on its length along $\mathrm{z}$ as known.

Let us review and discuss the existing design formulas. The resonant length of the patch, however is

Corresponding Author: Zuhair M. Hejazi, Department of Telecommunication Engineering, Faculty for Engineering Technology, Yarmouk University, Irbid, Jordan, hejazi@yu.edu.jo, +962-2-7211111 


\section{J. Eng. Applied Sci., 14 (Supplimentary 01): 8548-8558, 2019}

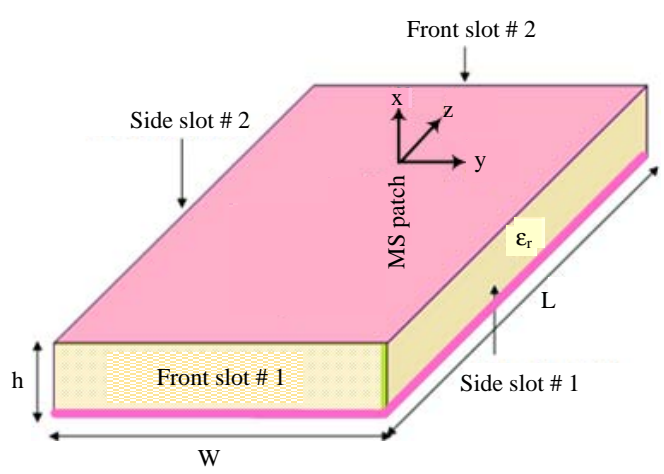

Fig. 1: A typical geometry of a rectangular MS patch antenna

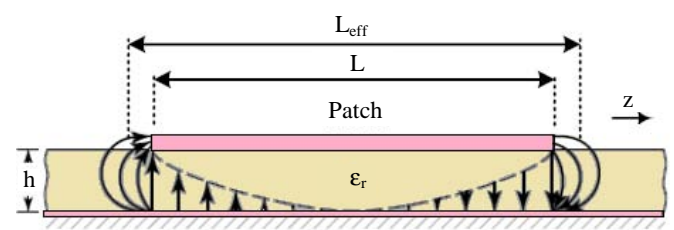

Fig. 2: A side-view of the MS patch antenna showing the length extension due to fringing effect

not exactly equal to the physical length, due to what is known as the fringing effect. It makes the effective electrical length of the patch longer than its physical length $\mathrm{L}_{\text {eff }}>\mathrm{L}$. Thus, the resonance condition depends on $\mathrm{L}_{\text {eff }}$ not $\mathrm{L}$ as illustrated in Fig. 2 and Eq. 1:

$$
\mathrm{f}_{\mathrm{r}}=\frac{\mathrm{c}}{2 \mathrm{~L}_{\text {eff }} \sqrt{\varepsilon_{\text {reff }}}}
$$

Where:

$\mathrm{c}=$ The speed of light in free space

$\varepsilon_{\mathrm{reff}}=$ Computed as Constantine (2005)

$$
\varepsilon_{\text {reff }}=\frac{\varepsilon_{\mathrm{r}}+1}{2}+\frac{\varepsilon_{\mathrm{r}}-1}{2}\left(1+12 \frac{\mathrm{h}}{\mathrm{W}}\right)^{-1 / 2} \quad \frac{\mathrm{W}}{\mathrm{h}}>1
$$

where, $\mathrm{L}_{\text {eff }}=2 \Delta \mathrm{L}$ and $\Delta \mathrm{L}$ is the length extension on each slot end which is a function of $\varepsilon_{\text {reff }}$ and the ratio $(\mathrm{W} / \mathrm{h})$. A very popular approximate relation for the length extension is given:

$$
\Delta \mathrm{L}=0.412 \mathrm{~h} \frac{\left[\varepsilon_{\mathrm{reff}}+0.3\right][(\mathrm{W} / \mathrm{h})+0.264]}{\left[\varepsilon_{\mathrm{reff}}+0.258\right][(\mathrm{W} / \mathrm{h})+0.8]}
$$

Using Eq. 3 for the resonant frequency of the dominant $\mathrm{TM}_{010}$ mode with and without the fringing effect, a fringe factor is found by Constantine (2005) as follows:
With the fringe effect:

$$
\mathrm{f}_{\mathrm{r}(\text { fringe })}=\frac{\mathrm{c}}{2 \mathrm{~L}_{\text {eff }} \sqrt{\varepsilon_{\text {reff }}}}
$$

Without the fringe effect:

$$
\mathrm{f}_{\mathrm{r}}=\frac{\mathrm{c}}{2 \mathrm{~L} \sqrt{\varepsilon_{\mathrm{r}}}}
$$

A fringe factor is then:

$$
\mathrm{q}=\frac{\mathrm{f}_{\mathrm{r}(\text { fringe })}}{f_{\mathrm{r}}}=\frac{L}{L_{\text {eff }}} \sqrt{\frac{\varepsilon_{\mathrm{r}}}{\varepsilon_{\text {reff }}}}
$$

It is also known that increasing the substrate thickness $h$, increases the surface waves resulting in unwanted coupling. The width of a patch antenna is known to be found from Constantine (2005):

$$
\mathrm{W}=\frac{\mathrm{c}}{2 \mathrm{f}_{\mathrm{r}}} \sqrt{\frac{2}{\left(\varepsilon_{\mathrm{r}}+1\right)}}
$$

The assumption in the model, at the dominant TM mode, leads to the assumption of a uniform field distribution along the width and $\mathrm{h}$ and that the side slots do radiate weakly in the principal E- and H-planes, i.e., their field intensity might be small compared to that radiated by the front slots $\# 1$ and $\# 2$. However, it is known that there is an undesired isolation coupling between adjacent patches in any array, believed to occur between the patch side slots. This undesired coupling may result in parasitic capacitances which might contribute to lowering the resonant frequency.

Also, from Eq. 4-7, the quantities $\varepsilon_{\text {reff }}$ and $\Delta \mathrm{L}$ are all depending on the $\mathrm{W} / \mathrm{h}$, not only $\mathrm{h}$, implying that the resonant frequency might be affected by the patch widths and the coupling between them as well. If, so, a coupling factor, similar to the fringe factor might be taken into account. Thus, a way to reduce or compensate both fringing effect and the isolation coupling might be considered in the design with keeping a compact size of the array if possible.

In a recent research by Hejazi and Al-Zoubi (2019), the fringing effects on resonant frequency and performance of a single MS patch antenna was investigated in detail by applying a novel corrugated structure. Very promising results were achieved. However, the isolation coupling issue, in particular and the antenna overall performance in general, cannot be verified with a single corrugated patch. 
Generally, it was found by Hejazi and Al-Zoubi (2019) that corrugating the front patch slots would compensate the length extension $\Delta \mathrm{L}$ to some extent while corrugating the side patch slots would reduce the isolation coupling in case of adjacent patch. It is well-known that such a coupling can also be easily reduced, if the separation between the patches is increased which on the other hand, would greatly increase the array size.

In a previous research by Hejazi and Jiang (2005), Hejazi and Ali (2014), Hejazi and Omar (2005) and Hejazi et al. (2008) on Ultra-Narrowband (UNB) passband filters, the issue of greatly reducing the inter-resonator coupling was a key to achieve UNB filtering i.e., if, the inter-resonator coupling of a filter is forced to occur in regions with minimum current strength, it can be expected to have a filter with extremely small coupling coefficient, hence, an UNB performance. This was achieved by pushing away the current peaks of the resonators from each other without increasing the resonator separation. The novel proposed approach of corrugating the patch edges might achieve a similar effect on the undesired isolation coupling between adjacent patches and the fringing effect as well.

\section{MATERIALS AND METHODS}

Isolation coupling planes of 2-element MS array: It is known that for two rectangular microstrip patches, the coupling for two side-by-side elements is a function of the relative alignment as Constantine (2005), Pozar and Kaufman (1987) and Pozar (2004) i.e., when the elements are positioned collinearly along the E-plane, referred
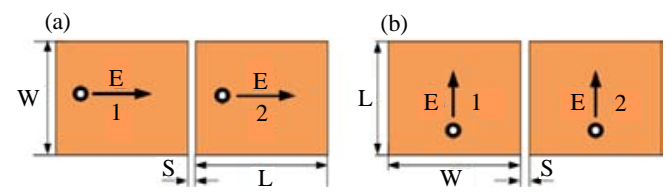

Fig. 3: (a, b) E- and H-plane coupling arrangements of MS patch array antennas for 2-elements

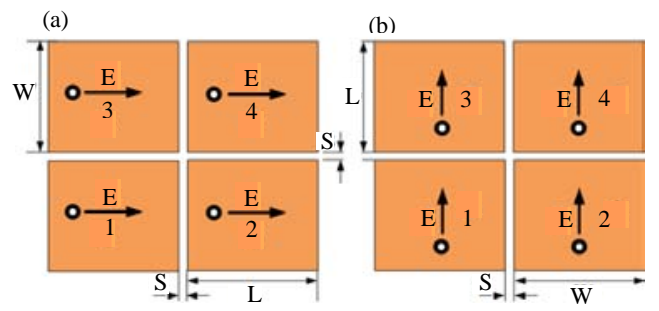

Fig. 4: (a, b) E- and H-plane coupling arrangements of MS patch array antennas for 4-elements to as the E-plane as shown in Fig. 3a when they collinearly along the $\mathrm{H}$-plane, referred to as the $\mathrm{H}$-plane as shown in Fig. 3b.

It is also known that, for an edge-to-edge separation of $\mathrm{S}$, the E-plane demonstrates the smallest isolation coupling for very small spacing. However, it should be noted that the spacing, at which one plane coupling dominates the other one, depends on the electrical properties and dimensions of the MS patch antenna as Constantine (2005).

Isolation coupling planes of 4-element array: Close observation of the 4-element arrangement in Fig. 4a, one can see that the 2 horizontal elements in each row are E-plane while the 2 vertical elements in each column are $\mathrm{H}$-plane coupled. In Fig. 4b, the opposite is observed. So, an array of either type consists of both $\mathrm{E}$ - and $\mathrm{H}$-plane arrangements.

In an array of 2 or more elements arranged only in a horizontal row of E-plane arrangement as in Fig. 3a, the undesired coupling may occur only between the front slots while in H-plane arrangement, it may occur only between the side slots (Fig. 3b).

In the horizontal-vertical arrangement of either $\mathrm{E}$ - or $\mathrm{H}$-plane as in Fig. 4a, b, one can expect the undesired couplings to occur in both front and side-slots of the patches. So, the effect of corrugating these slots, depending on the coupling strength regions and type of arrangement, might be investigated to find out the best configuration for optimum performance. This is done in the next sections.

\section{RESULTS AND DISCUSSION}

Simulation results of 2-element array-Example design specifications: To verify these considerations and prove the concept on the effects of the corrugation location and depth of the array edges on the isolation coupling, resonant frequency, radiation pattern, gain and cross polarization, we choose for simulations a 2-element patch array composed of standard square patches as a reference, each fed by a coax probe, then, compare any changes in performance with applying different corrugation shapes and depths.

The dimensions of the reference MS patches, used in the array is $40 \times 40 \mathrm{~mm}$, placed on a Rogers RT/Duroid 5870 substrate with $\varepsilon_{\mathrm{r}}=2.33, \mathrm{~h}=0.51 \mathrm{~mm}$ and $\tan \delta=0.0012$. The geometry of the single patch antenna, used in the 2-element array for simulations is shown in Fig. 5a. Coaxial feeding parameters: inner conductor radius 


\section{J. Eng. Applied Sci., 14 (Supplimentary 01): 8548-8558, 2019}
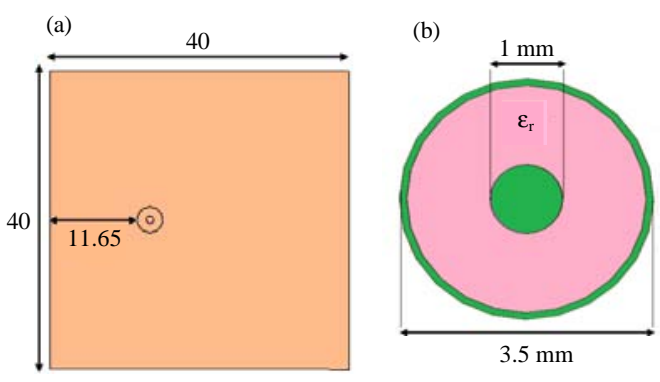

Fig. 5: a) Geometry of the reference square patch antenna and b) Coaxial feeding

$0.5 \mathrm{~mm}$, outer conductor $1.75 \mathrm{~mm}$, Teflon based dielectric with $\varepsilon_{\mathrm{r}}=2.08$ as in Fig. 5b. Feeding position: $11.65 \mathrm{~mm}$ from the edge for all cases. Corrugation depths and their dimensions might be $<<\lambda_{g}$, i.e., the guided wavelength.

In the next sections, full wave EM simulations are carried out using Sonnet (Method of Moments-MoM) by Anonymous (2017) and HFSS (Finite Element Method- FEM) software by Anonymous (2008).

The reference 2-element array in the $\mathrm{E}$ - and $\mathrm{H}$-planes: Conventionally known, if the spacing $\mathrm{S}$ is increased, one expects the isolation coupling factor $\left(\left|\mathrm{S}_{21}{ }^{2}\right|\right)(\mathrm{dB})$ would decrease accordingly on the cost of greatly increasing the array size. The simulations for this well-known approach are not shown here. In summary, the decrease of the coupling in the E-plane arrangement is about $(-16.3 \mathrm{~dB})$, the gain is $6.86 \mathrm{dBi}$ and the cross polarization level is $-24 \mathrm{~dB}$. In contrast, for the $\mathrm{H}$-plane arrangement, the peak gain is $7.45 \mathrm{dBi}$ which is about $0.6 \mathrm{~dB}$ higher than E-plane arrangement and the cross polarization level is somewhat better, i.e., $-26 \mathrm{~dB}$.

The proposed corrugated 2-element E-plane array: Firstly, the simulations are carried out for front-corrugated 2-element array in the E-plane arrangement, then compared with the reference patch array using a fixed separation of $1 \mathrm{~mm}$ for all cases.

The $S_{21}$ responses are compared in Fig. 6 using Sonnet and HFSS with corrugation depths of 2.5 and $5 \mathrm{~mm}$.

From Fig. 6, it is observed that the front-corrugated 2-element array with a depth of $2.5 \mathrm{~mm}$ reduces the isolation coupling by $1.8 \mathrm{~dB}$ only. Increasing the depth to $5 \mathrm{~mm}$, the additional reduction is slight, i.e., only $0.2 \mathrm{~dB}$. However, Fig. 7 and 8 show that the gains are 7.25 and $7.28 \mathrm{dBi}$ for 2.5 and $5 \mathrm{~mm}$ depths, respectively. The cross polarization level is much better, i.e., $-38 \mathrm{~dB}$.

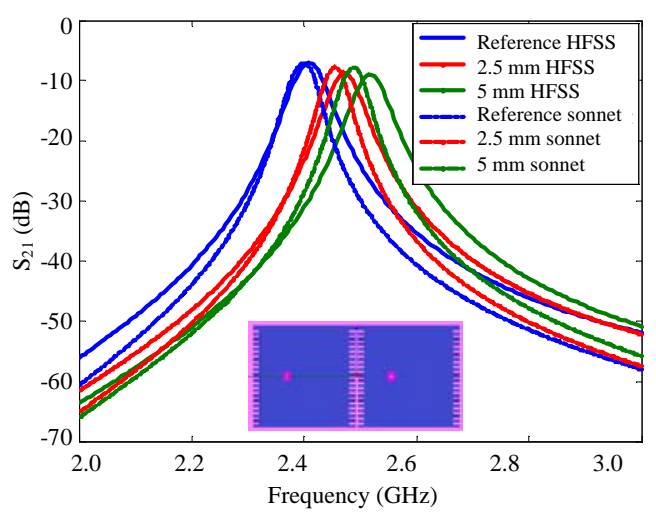

Fig. 6: Comparison of the coupling isolation between 2-element E-plane reference patch array with fronts-corrugated array

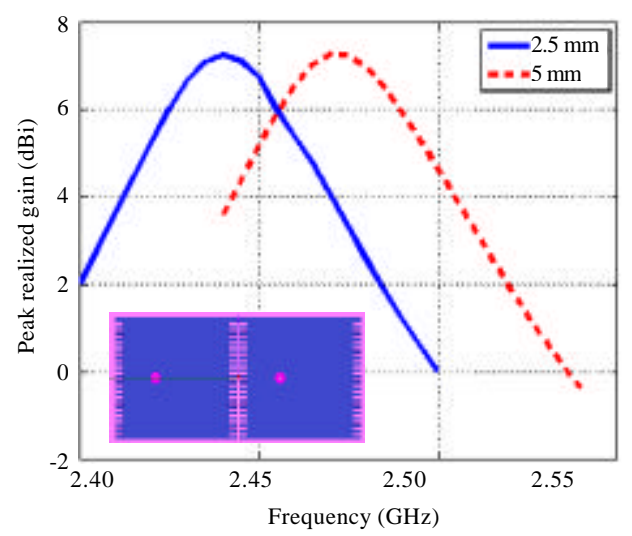

Fig. 7: Comparison of the peak realized gains of the E-plane 2-element fronts-corrugated array at 2.5 and $5 \mathrm{~mm}$ depths

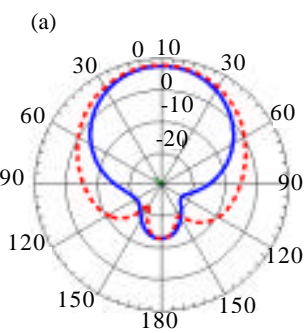

(b)

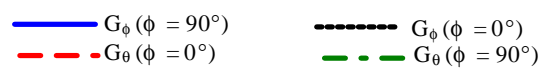

Fig. 8: (a, b) 2D radiation patterns of the E-plane 2-element fronts-corrugated array

So, adding front corrugations to the radiating slots of the patches increase the gain and reduces the cross polarization level while the effect on the coupling is slight. 


\section{J. Eng. Applied Sci., 14 (Supplimentary 01): 8548-8558, 2019}

In general, all the other explored shapes in the E-plane arrangement of the array (not presented, here, due to space limit) show slight effects on the coupling, thus, we might also explore all these shapes in the $\mathrm{H}$-plane arrangement. This is done next among others.

Corrugated 2-element array in the H-plane: The basic arrangement of Fig. 3b with corrugated 2-elelment array in the H-plane is now simulated using sonnet and HFSS. The frequency responses are compared with the reference array in Fig. 9 for fronts-corrugation depths of 2.5 and $5 \mathrm{~mm}$. In this case, there is almost no effect on the isolation coupling but a slight shift-up of the center frequency.

However as shown in Fig. 10 and 11, the peak gain is $7.7 \mathrm{dBi}$ for both corrugation depths which is about $0.3 \mathrm{~dB}$ higher than the E-plane fronts-corrugated arrangement and the H-Plane reference array. The cross polarization level is very low $(-38 \mathrm{~dB})$.

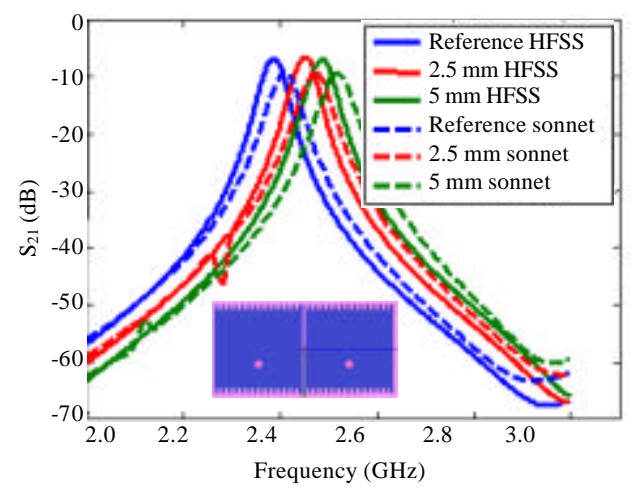

Fig. 9: Comparison of the coupling isolation between 2-element H-plane reference patch array with fronts-corrugated array

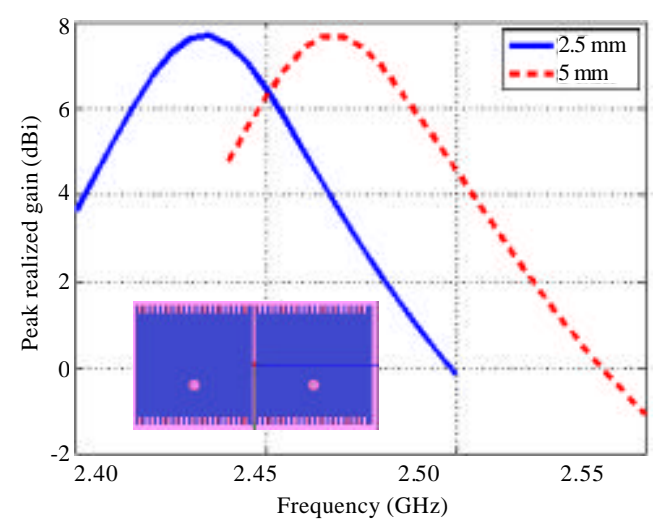

Fig. 10: Comparison of peak realized gains of the 2-element $\mathrm{H}$-planefronts-corrugated array
Figure 12 shows a comparison of the coupling isolation between 2-Element H-plane reference patch array with sides-corrugated array at depths of 2.5 and $5 \mathrm{~mm}$. A strong effect on the coupling is observed.

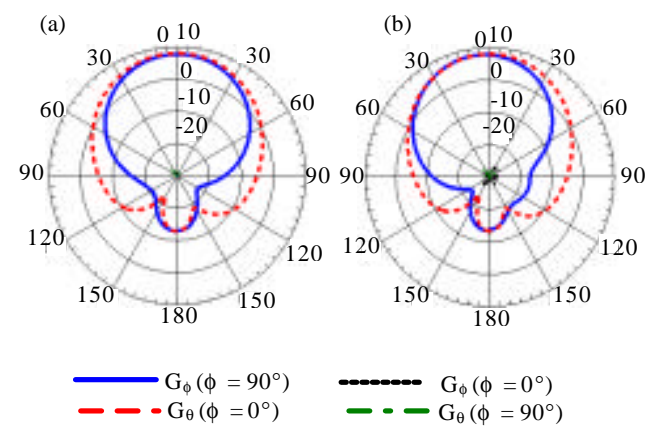

Fig. 11: 2D radiation patterns of the 2-element H-plane fronts-corrugated array: a) $2.5 \mathrm{~mm}$ and b) $5 \mathrm{~mm}$

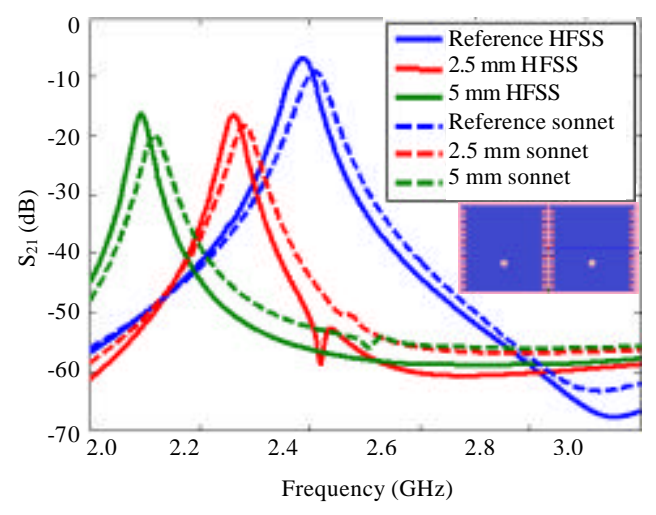

Fig. 12: Comparison of the coupling isolation between 2-element $\mathrm{H}$-plane reference patch array with sides-corrugated array

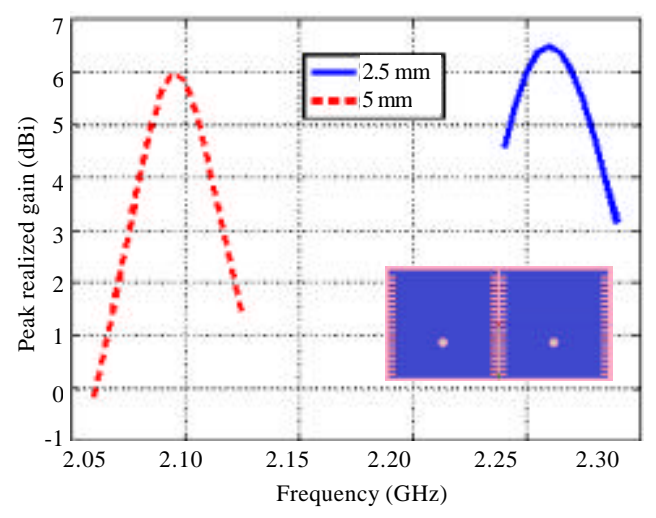

Fig. 13: Comparison of the peak realized gain of the 2-element $\mathrm{H}$-plane reference patch array with sides-corrugated array 


\section{J. Eng. Applied Sci., 14 (Supplimentary 01): 8548-8558, 2019}

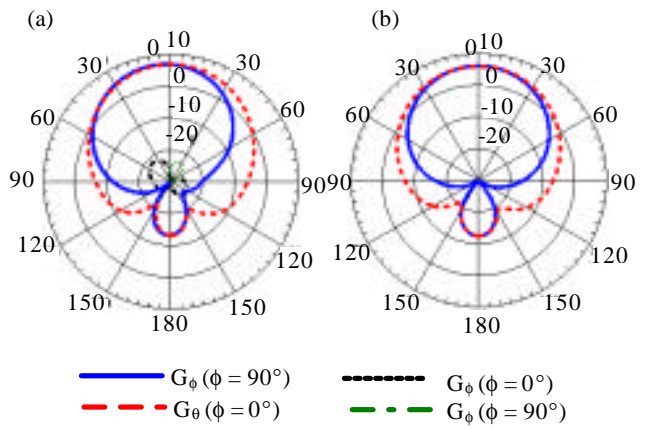

Fig. 14: 2D radiation pattern of the 2-element $\mathrm{H}$-plane reference patch array with sides-corrugated array: a) $2.5 \mathrm{~mm}$ and b) $5 \mathrm{~mm}$

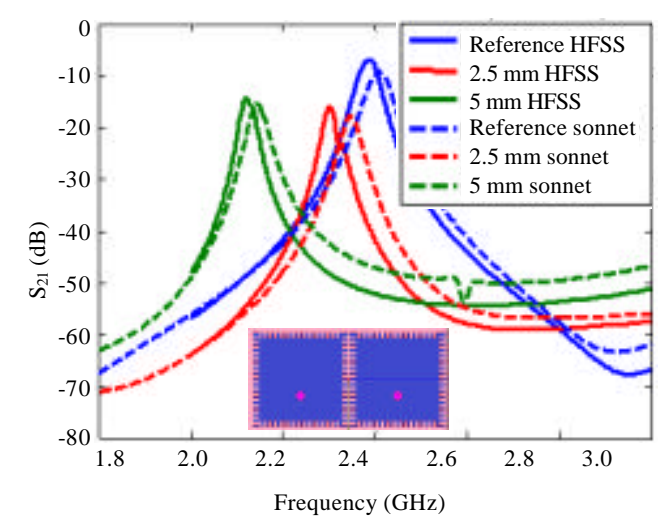

Fig. 15: Comparison of the coupling between 2-element $\mathrm{H}$-plane reference patch array with an all-sides-corrugated array

i.e., a coupling reduction of about $(-9 \mathrm{~dB})$ with a depth of $2.5 \mathrm{~mm}$ and $(-11 \mathrm{~dB})$ with $5 \mathrm{~mm}$ depth. The gains, however are slightly reduced to 6.5 and $6 \mathrm{dBi}$ for 2.5 and $5 \mathrm{~mm}$ depths, respectively as shown in Fig. 13. The cross polarization level is still very low $(-30 \mathrm{~dB})$ and the radiation pattern is symmetric as shown in Fig. 14. Thus, sides-corrugated array in the H-plane arrangement may be recommended to reduce the undesired coupling and the cross polarization on the cost of slight reduction of the gain, unless a better improvement is obtained with other corrugation shapes.

The all-sides-corrugated array of the 2-element $\mathrm{H}$-plane arrangement has also a relatively strong effect on the coupling reduction but less than that of the sides-corrugated array as shown in Fig. 15 , i.e., $8.6 \mathrm{~dB}$ for $2.5 \mathrm{~mm}$ depth and $6.4 \mathrm{~dB}$ for $5 \mathrm{~mm}$ depth. The down-shifts of the frequency are also, relatively high. The peak gains are 7.06 and $5.6 \mathrm{dBi}$ for 2.5 and $5 \mathrm{~mm}$ depths, respectively as shown in Fig. 16. However, the cross

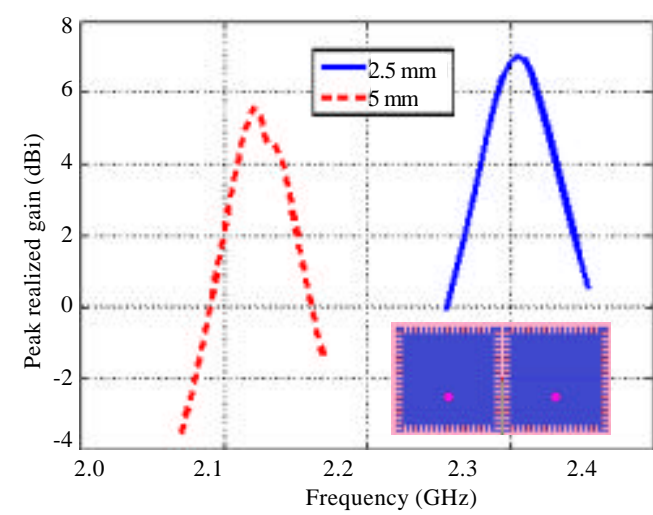

Fig. 16: Comparison of the peak realized gains of the 2-element $\mathrm{H}$-plane all-sides-corrugated array

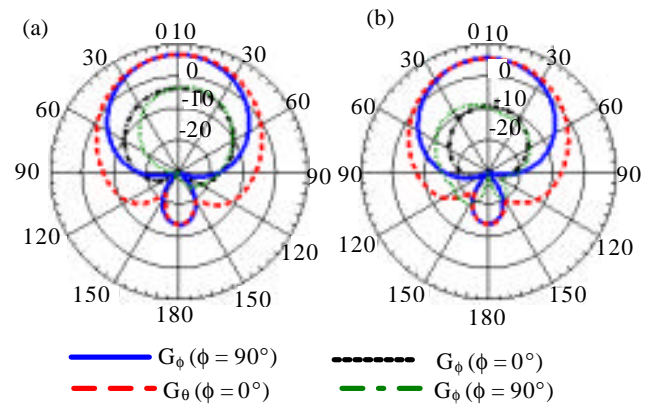

Fig. 17: 2D radiation pattern of the 2-element H-plane reference patch array with all-sides-corrugated array: a) $2.5 \mathrm{~mm}$ and b) $5 \mathrm{~mm}$

polarization level is deteriorated and equals to $(-10 \mathrm{~dB})$ and $(-15 \mathrm{~dB})$ for both corrugation depths as can be seen from Fig. 17.

Alternative corrugation shapes are now explored, such as: Cup-Cap, Cap-Cup, Cap-Cap and Cup-Cup in the $\mathrm{H}$-plane arrangement.

We start with the Cup-Cap corrugation shape (un), then the Cap-Cup shape (nu). Finally, the Cap-Cap(nn) and Cup-Cup( $\cup \cup)$ shapes are all investigated and compared.

It should be noted that the corrugation shapes of two patches might always be symmetric in some way, to ensure acceptable matching between them, i.e., $S_{11}$ and $S_{22}$ to fit on the same center frequency of $\mathrm{S}_{21}$ and $\mathrm{S}_{12}$. Also, symmetric radiation patterns might be achieved.

The simulation results are summarized and compared with the reference array in Fig. 18 for $2.5 \mathrm{~mm}$ depth. The Cup-Cap and Cap-Cup $S_{21}$ responses overlap (thus, not both seen in the figure), except that the $\mathrm{S}_{11}$ of Cap-Cup is $16 \mathrm{~dB}$ better than the Cup-Cap shape (also not shown). 


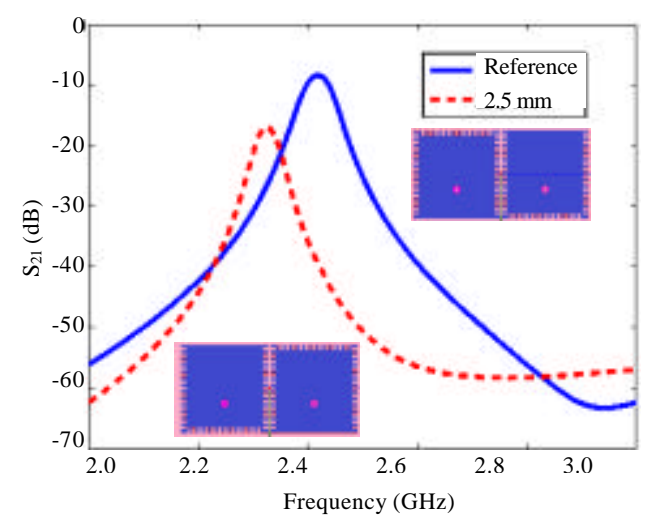

Fig. 18: Comparison of $\mathrm{S}_{21}$ of the H-plane reference patch array with both Cup-cap and Cap-cup corrugations

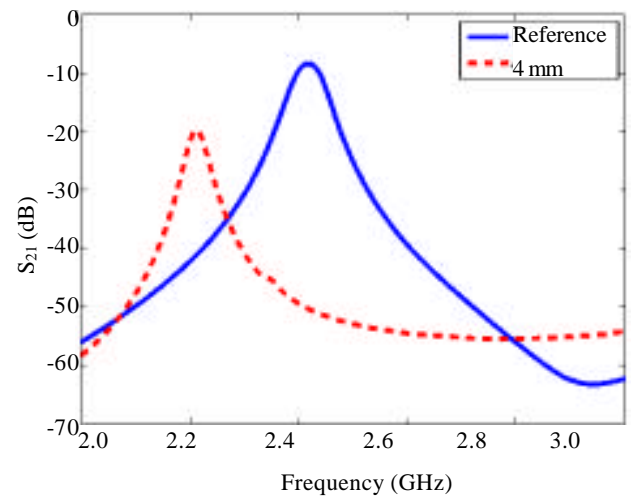

Fig. 19: H-plane Cap-Cup and (Cup-Cap response is not shown as it overlaps)

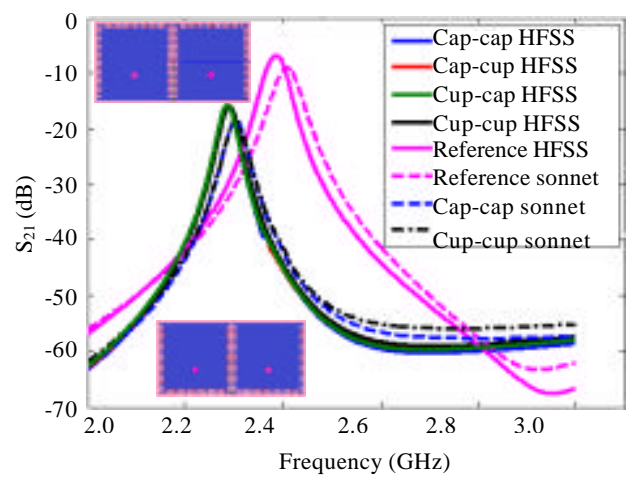

Fig. 20: Comparison of S21 responses of the Cap-Cap and Cup-Cup shapes (at $2.5 \mathrm{~mm}$ depth) with the reference array

The coupling reduction compared with the reference array is impressive $(8.5 \mathrm{~dB})$ but slightly less than the side-corrugated shape presented in Fig. 12. Further,

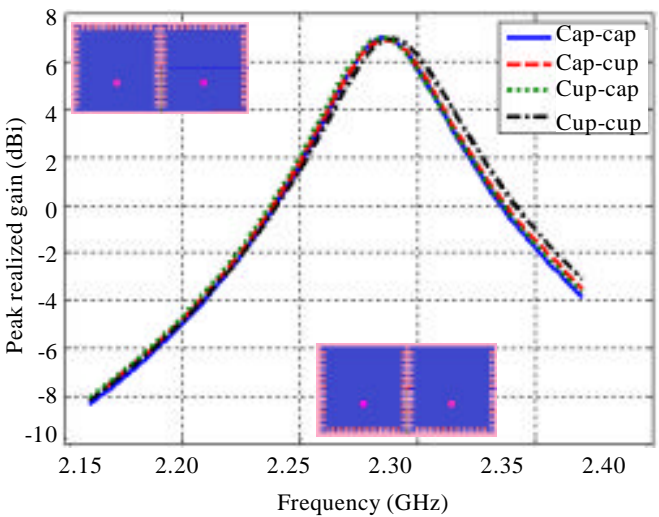

Fig. 21: Comparison of the peak realized gains of the 2-element H-plane Cap-Cap, Cap-Cup, Cup-Cap and Cup-Cup corrugation shapes (at $2.5 \mathrm{~mm}$ depth)

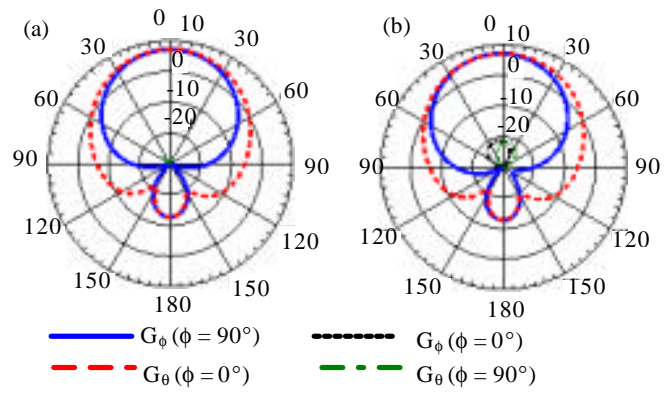

Fig. 22: 2D radiation patterns of the 2-element H-plane corrugated shapes: a) Cap-Cap and b) Cap-Cup

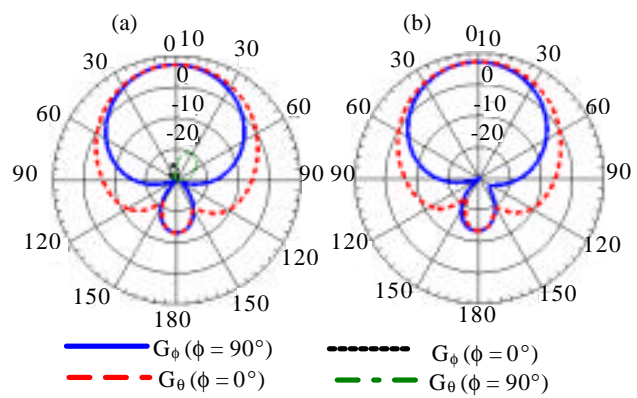

Fig. 23: 2D radiation patterns of the 2-element $\mathrm{H}$-plane corrugated shapes: a) Cup-Cap and b) Cup-Cup

improvement is observed with the Cap-Cup shape having $4 \mathrm{~mm}$ corrugation depth as shown in Fig. 19. The improvement here, compared to the reference is $10.7 \mathrm{~dB}$, i.e., almost $2.2 \mathrm{~dB}$ more than that with $2.5 \mathrm{~mm}$ depth. The $\mathrm{H}$-plane Cap-Cap and Cup-Cup shapes are simulated and their $\mathrm{S}_{21}$ responses are compared in Fig. 20 with corrugation depths of $2.5 \mathrm{~mm}$. In comparison to reference array, improvements of 9.3 and $11 \mathrm{~dB}$, respectively are observed. It can be noticed from Fig. 21-23 that all four 
arrangements have almost the same gain of about $7 \mathrm{dBi}$ with a slight shift of frequency of about $10 \mathrm{MHz}$. The cross polarization levels are $(-40,-30,-30$ and $40 \mathrm{~dB})$ for Cap-Cap, Cap-Cup, Cup-Cap and Cup-cup, respectively.

So, the H-plane Cap-Cap and Cup-Cup shapes have the best minimum cross polarization and relatively good gain, besides a good coupling reduction.

Current distributions of 2-element array: The current distributions on the 2-element patch arrays of the various arrangements would provide an additional insight on the corrugation effects, their locations and depths in comparison with the reference patch array.

Current distributions of 2-element E-plane array: In the E-plane arrangements (not shown here due to space limit),

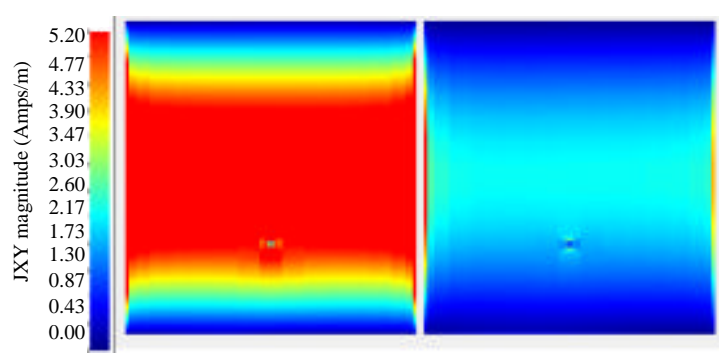

Fig. 24: Current distribution of the reference H-plane 2-element array

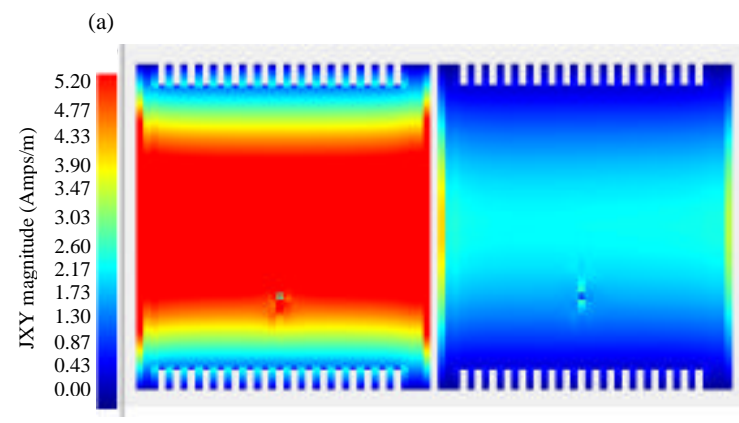

(b)

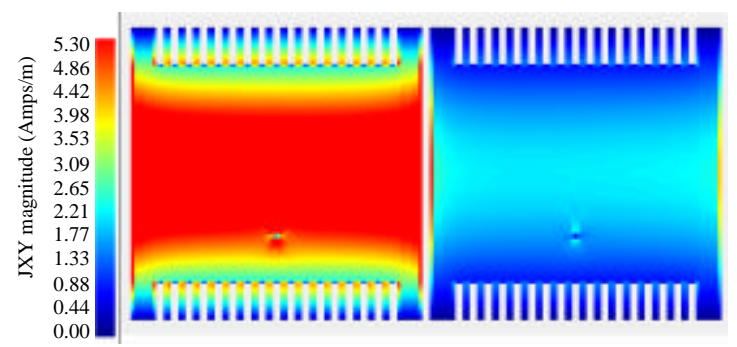

Fig. 25: Current distribution of H-plane front-corrugated 2-elements with depths of: a) $2.5 \mathrm{~mm}$ and b) $5 \mathrm{~mm}$ it is observed that the current peaks are on the patch sides while the fronts have less current crowding. So, the isolation coupling may be stronger on the columns of a multi-element array. Also, the second patch in the corrugated E-plane arrays is relatively strong-coupled, compared to that of the reference array.

Current distributions of 2-element $\mathrm{H}$-plane array: The current distributions of key simulated cases above in the H-plane arrangements with different corrugation depths and locations are presented next (all at resonance).

Figure 24 shows the current distribution on the reference H-plane 2-element array for comparisons. Figure $25 \mathrm{a}, \mathrm{b}$ on the fronts-corrugated shapes while Fig. 26, on the sides-corrugated array.

The current distribution on the H-plane all-sidescorrugated array is shown in Fig. 27a, b. The current distributions on the alternative Cap-Cup, Cap-Cap and Cup-Cup corrugation shapes are shown as indicative samples in Fig. 28-30, respectively. All are with corrugation depths of $4 \mathrm{~mm}$.

In the H-plane arrangements, it can be seen that the current peaks are also, on the patch sides while the fronts have less current crowding, so, the isolation coupling might be stronger on the rows of a multi-element array. It can also be observed that the second patch in the corrugated arrays is relatively weak-coupled, compared to that of the reference array, especially, those from Fig. 26-30.
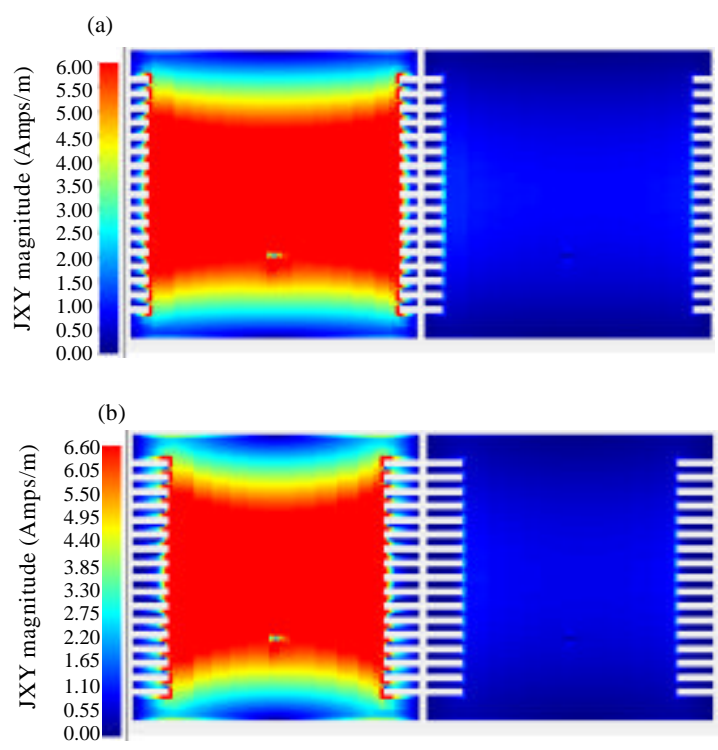

Fig. 26: Current distribution of H-planesides-corrugated 2-elements with depths of: a) $2.5 \mathrm{~mm}$ and b) $5 \mathrm{~mm}$ 


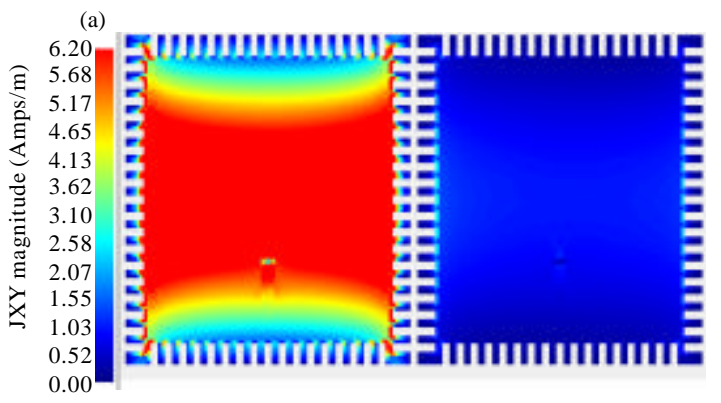

(b)

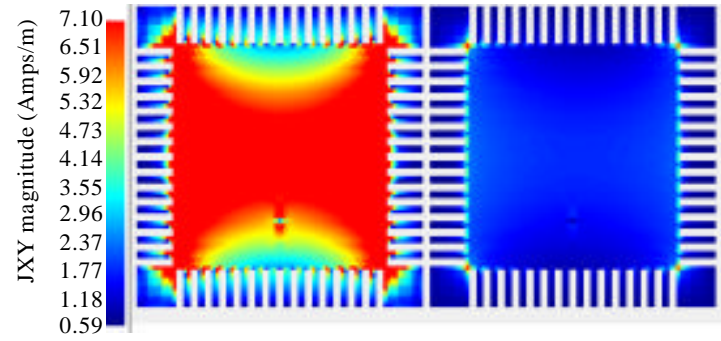

Fig. 27: Current distribution of H-plane all-sidescorrugated 2-elements with depths of: a) $2.5 \mathrm{~mm}$ and b) $5 \mathrm{~mm}$

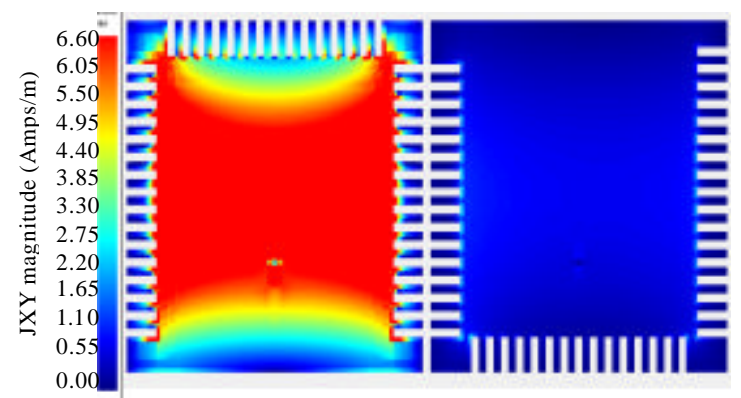

Fig. 28: Current distribution of H-plane Cap-Cupcorrugated 2-elements with depths of $4 \mathrm{~mm}$

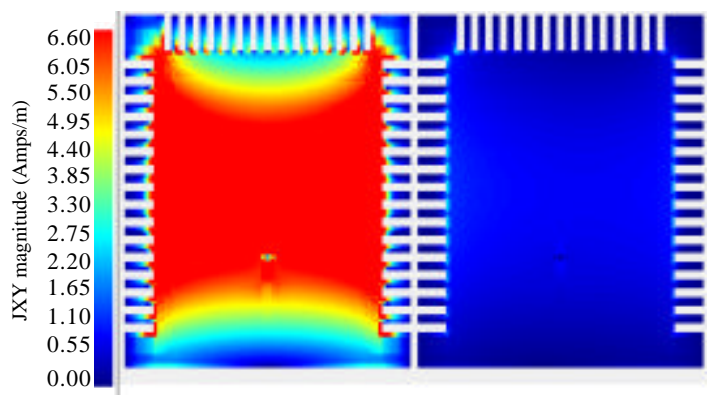

Fig. 29: Current distribution of H-plane Cap-Capcorrugated 2-elements with the depths of $4 \mathrm{~mm}$

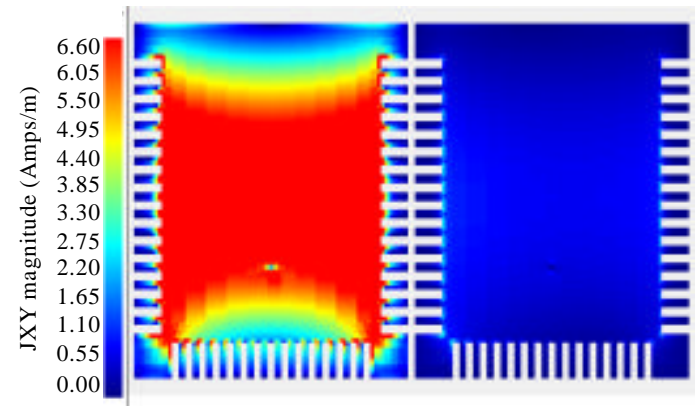

Fig. 30: Current distribution of H-plane Cup-Cupcorrugated 2-elements with depths of $4 \mathrm{~mm}$. This is the best coupling reduction

\section{CONCLUSION}

A new class of corrugated MS patch antenna arrays was investigated in detail using various corrugated structures with different depths. The 2-element arrays of these structures were compared with a standard reference (un-corrugated) array in both the E- and H-plane arrangements. The emphasis was on ways to reduce the undesired isolation coupling and the effects on the overall antenna performance while maintaining the same compact size in all cases. In the E-plane arrangements, fronts-corrugated structures showed slight improvements of the isolation coupling with apeak realized gain of (7.3 $\mathrm{dBi}$ ) but showed excellent improvement of the cross polarization level $(\mathrm{X}-\mathrm{Pol}=-38 \mathrm{~dB})$. In the $\mathrm{H}$-plane arrangements, however, the fronts-corrugated array had also a slight effect on the coupling but increased the peak gain to $(7.7 \mathrm{dBi})$ with the same X-Pol $(-38 \mathrm{~dB})$ as the E-plane. However with sides-corrugated array, it was observed a strong effect on the coupling, i.e., a reduction of up to $(-11 \mathrm{~dB}$ below the reference array) on the cost of a slight reduction of the gain $(6.5 \mathrm{dBi})$ and X-Pol $(-11 \mathrm{~dB})$. Also, in the H-plane Cup-Cap and Cap-Cup shapes, the coupling reduction was impressive (up to $-7.10 \mathrm{~dB}$ below the reference), a gain of $(7 \mathrm{dBi})$ and X-Pol of $(-30 \mathrm{~dB})$ which is still very good, compared to the reference array. The H-plane Cap-Cap and Cup-Cup shapes showed the best minimum X-Pol $(-40 \mathrm{~dB})$ and a relatively good gain $(7 \mathrm{dBi})$, besides an impressive coupling reduction of $(-9.3 \mathrm{~dB})$ and $(-11 \mathrm{~dB}$ below the reference), respectively. The proposed novel 2-element corrugated structures can be used as building blocks to construct multi-element arrays. If the corrugations are properly controlled and optimized in locations and depths, the novel structures with improved performance, may are very useful in many critical applications. 


\section{SIGNIFICANCE STATEMENT}

In this research, the emphasis is focused on improving the overall performance including the isolation coupling between 2-element MS patch array antenna as a building block for a new class of multi-element corrugated array, i.e., how to reduce the undesired isolation coupling and improve the overall antenna performance at the same time, without affecting the antenna compact size. This is done by carefully exploring which sides of the array to corrugate and their depths. The effects of several proposed structures with different E-and H-planes arrangements of the array are investigated and compared in detail. Most of the proposed corrugated structures, greatly reduce the cross polarization levels to almost zero. Some of them reduce also the coupling to impressive levels besides the gain to some extent. If the corrugations are properly controlled and optimized in locations and depths, the novel corrugated structures pave the way for a new class of MS multi-element patch array antennas which may be very useful in many critical applications requiring robust, lower profile, high performance low cost antennas.

\section{ACKNOWLEDGEMENT}

The researchers would like to thank the IFERP, the JEAS reviewers and the editorial team for their valuable comments and suggestions in enhancing this research article.

\section{REFERENCES}

Anonymous, 2017. Simulation software based on moment method. Sonnet Software, Syracuse, New York, USA.

Anonymouse, 2008. HFSS: High frequency structure simulator based on the Finite Element Method. Version 11.1, ANSYS Corporation, Canonsburg, Pennsylvania, USA.

Casu, G., C. Moraru and A. Kovacs, 2014. Design and implementation of microstrip patch antenna array. Proceedings of the 2014 10th International Conference on Communications (COMM'14), May 29-31, 2014, IEEE, Bucharest, Romania, pp: 1-4.

Constantine, A.B., 2005. Antenna Theory: Analysis and Design. 3rd Edn., John Wiley \& Sons, Hoboken, New Jersey, USA., ISBN-13:978-0-471-66782-7, Pages: 1136.

Gour, P., R. Mishra, A.S. Zadgaonkar andP.K. Sahu, 2017. Circular polarized broad band microstrip patch antenna for C band. Intl. J. Comput. Technol. Appl., 9: 191-197.
Hejazi, Z.M. and A. Omar, 2005. Modeling and simulation of novel ultra-narrowband miniature microstrip filters for mobile and wireless critical applications. Microwave Opt. Technol. Lett., 45: 35-39.

Hejazi, Z.M. and A.S. Al-Zoubi, 2019. Novel corrugated microstrip patch antenna: Effects on resonant frequency, coupling and cross polarization. Proceedings of the 20196 th International Conference on Electrical and Electronics Engineering (ICEEE'19), April 16-17, 2019, IEEE, Istanbul, Turkey, ISBN:978-1-7281-3911-1, pp: 173-179.

Hejazi, Z.M. and Z. Ali, 2014. Multiband bandpass filters with suppressed harmonics using a novel defected ground structure. Microwave Opt. Technol. Lett., 56: 2726-2731.

Hejazi, Z.M. and Z. Jiang, 2005. A new model to calculate the coupling coefficient for more accurate filter design and further development of narrowband-filter performance. Microwave Opt. Technol. Lett., 47: 180-185.

Hejazi, Z.M., M.C. Scardelletti, F.W. van Keuls, A.A. Omar and A.S. Al-Zayed, 2008. EM full-wave analysis and testing of novel quasi-elliptic microstrip filters for ultra narrowband filter design. Prog. Electromagnet. Res., 85: 261-288.

Khan, M.U., M.S. Sharawi and R. Mittra, 2015. Microstrip patch antenna miniaturisation techniques: A review. IET. Microwaves Antennas Propag., 9: 913-922.

Kumar, S., A. Sharma, B.K. Kanaujia, M.K. Khandelwal and A.K. Gautam, 2016. Dual-band stacked circularly polarized microstrip antenna for $\mathrm{S}$ and $\mathrm{C}$ band applications. Intl. J. Microwave Wirel. Technol., 8: 1215-1222.

Lee, H.R., H.K. Ryu, S. Lim and J.M. Woo, 2009. A miniaturized, dual-band, circularly polarized microstrip antenna for installation into satellite mobile phones. IEEE. Antennas Wirel. Propag. Lett., 8: 823-825.

Li, Q., F. S. Zhang, G. Zhang, B. Wang and M. Liang, 2014. A single-feed dual-band dual-sense circularly polarized microstrip antenna. Prog. Electromagnet. Res., 51: 27-33.

Pozar, D.M. and B. Kaufman, 1987. Increasing the bandwidth of a rectangular microstrip antenna by proximity coupling. Electr. Lett., 23: 368-369.

Pozar, D.M., 2004. Microwave Engineering. 3rd Edn., Wiley, Hoboken, New Jersey, USA., ISBN:9780471448785, Pages: 720.

Raj, T. and B. Chauhan, 2014. Single layer dual band microstrip patch antenna using probe feed. Intl. J. Comput. Appl., 92: 1-5. 
Rao, K.M.S. and M.V.S. Prasad, 2019. Design of microstip patch antennas at uwb for wireless applications. J. Eng. Appl. Sci., 14: 4728-4735.

Sharma, V. and M.M. Sharma, 2014. Dual band circularly polarized modified rectangular patch antenna for wireless communication. Radioengineering, 23: 195-202.
Singh, D.K., B.K. Kanaujia, S. Dwari, G.P. Pandey and S. Kumar, 2015. Multiband circularly polarized stacked microstrip antenna. Prog. Electromagnet. Res. C., 56: 55-64.

Zakaria, N., S.K.A. Rahim, T.S. Ooi, K.G. Tan and A.W. Reza et al., 2012. Design of stacked microstrip dual-band circular polarized antenna. Radioengineering, 21: 875-880. 